\title{
Managing the pediatric patient with refractory asthma: a multidisciplinary approach
}

This article was published in the following Dove Press journal:

Journal of Asthma and Allergy

20 April 2017

Number of times this article has been viewed

\author{
James Cook ${ }^{1,2}$ \\ Fran Beresford ${ }^{2}$ \\ Valentina Fainardi' \\ Pippa Hall ${ }^{2}$ \\ Georgie Housley ${ }^{2}$ \\ Angela Jamalzadeh ${ }^{2}$ \\ Michelle Nightingale ${ }^{2}$ \\ David Winch ${ }^{2}$ \\ Andrew Bush ${ }^{1,2}$ \\ Louise Fleming ${ }^{1,2}$ \\ Sejal Saglani ${ }^{1,2}$ \\ 'Inflammation, Repair and \\ Development, National Heart and \\ Lung Institute, Imperial College \\ London, ${ }^{2}$ Respiratory Paediatrics, \\ Royal Brompton Hospital, London, UK
}

Correspondence: Sejal Saglani Respiratory Paediatrics, Imperial College London, 368 Sir Alexander Fleming Building, Exhibition Road, London, SW7

2AZ, UK

Tel +442075943167

Fax +442075943119

Email s.saglani@imperial.ac.uk

\begin{abstract}
Children with asthma that is refractory to high levels of prescribed treatment are described as having problematic severe asthma. Those in whom persistent symptoms result from a failure of basic asthma management are described as having "difficult asthma", while those who remain symptomatic despite these factors having been addressed are described as having "severe therapy-resistant asthma" (STRA). The majority of children have difficult asthma; asthma that is poorly controlled because of a failure to get the basics of asthma management right. Modifiable factors including nonadherence to medication, persistent adverse environmental exposures, and psychosocial factors often contribute to poor control in these patients. As our skill in identifying and addressing modifiable factors has improved, we have found that a progressively smaller proportion of our clinic patients is categorized as having true STRA, resulting in an infrequent resort to escalation of treatment. Many of the modifiable factors associated with the diagnosis of difficult asthma can be identified in a general pediatric clinic. Characterization of more complex factors, however, requires the time, skill, and expertise of multiple health care professionals within the asthma multidisciplinary team. In this review, we will describe the structured approach adopted by The Royal Brompton Hospital in the management of the child with problematic severe asthma. We highlight the roles of members of the multidisciplinary team at various stages of assessment and focus on prominent themes in the identification and treatment of modifiable factors.
\end{abstract}

Keywords: pediatric, asthma, multidisciplinary team

\section{Introduction}

One in every eleven children in the UK is currently prescribed treatment for asthma. ${ }^{1}$ The majority of these children experience satisfactory symptom control utilizing only low to moderate doses of inhaled corticosteroids. Approximately five percent, however, are prescribed higher doses, equivalent to steps 4/5 of the Global Initiative for Asthma guidelines $^{2}$ in an effort to control symptoms. This poorly controlled group represents a significant clinical challenge because of the associated morbidity and mortality, in addition to a disproportionately high utilization of health care resources. ${ }^{3}$ Furthermore, a long-term negative impact of severe childhood asthma on adult lung function and an association with chronic obstructive pulmonary disease (COPD) in later life has emerged. ${ }^{4}$

Children with on-going asthma symptoms despite maximal conventional treatments are described as having problematic severe asthma. ${ }^{5}$ Those in whom persistent symptoms result from a failure of basic asthma management are described as having "difficult asthma" (DA), while those who remain symptomatic despite these factors having been addressed are described as having "severe therapy-resistant asthma" 
(STRA). The key to adopting an appropriate and effective management strategy for a patient depends upon the correct distinction between DA and STRA. The proportion of children found to have modifiable factors affecting their asthma control is high. Over $50 \%$ of children with problematic severe asthma are categorized within the DA group after initial assessment. ${ }^{6}$ As our skills in identifying and addressing modifiable factors have improved, we have found that a progressively smaller proportion of patients are categorized as having true STRA resulting in infrequent escalation of treatment. It is important to emphasize that there remains a group of patients at significant risk of morbidity and mortality, even though they may not be prescribed high dose maintenance therapy. Seventy percent of asthma deaths described in the national review of asthma deaths (NRAD) 7 occurred in people who did not have "severe" asthma as defined by prescribed medication. However, asthma resulting in death is severe. It is therefore apparent that a better understanding of this high-risk group is required to refine who and how we classify severe asthma. ${ }^{8}$

Many of the modifiable factors associated with the diagnosis of DA can be identified in a general pediatric clinic. Characterization of more complex factors, however, requires the time, skill and expertise of multiple health care professionals including specialist respiratory nurses, physiotherapists, clinical psychologists, dietitians, clinical physiologists, safeguarding nurses, social workers, as well as doctors. The European Respiratory Society and American Thoracic Society recommend referral to a respiratory specialist when a child is prescribed high dose inhaled steroids $(800 \mu \mathrm{g} /$ day budesonide equivalent $)^{9}$ because specialized assessments and interventions are necessary to allow the identification and management of DA. Furthermore, the NRAD UK identified that $20 \%$ of asthma deaths were in patients who met the criteria for referral for specialist respiratory assessment, but had not been referred. ${ }^{7}$ It is important to note that there should be no complacency about the treatment of DA; it is not a benign condition, and death may result if modifiable factors are not rectified.

In this review, we will describe the structured approach adopted by The Royal Brompton Hospital (RBH) in the management of the child with problematic severe asthma. We will highlight the roles of members of the multidisciplinary team (MDT) at various stages of assessment and focus on prominent themes in the identification and treatment of modifiable factors. Steps one to three represent management that can be undertaken in a general pediatric clinic, while later steps require specialist input from the MDT. The benefits of this approach have been demonstrated in a retrospective review which identified that children diagnosed with DA (with modifiable factors identified and addressed) had significantly improved lung function, were on lower dose inhaled steroids, and had significantly fewer exacerbations up to 6 years after the assessment. ${ }^{10}$

\section{Step I: confirm diagnosis}

In cases where symptoms remain uncontrolled despite maximal guideline-recommended treatment, the possibility that there is an alternate diagnosis must be considered. Every child referred to RBH has an initial assessment by a consultant respiratory physician at which point evidence supporting a diagnosis of asthma is ascertained. Identification in the history of physician-confirmed wheeze and clinical improvement when using a bronchodilator are important. Objective measures supporting the key pathophysiological features of asthma, reversible airway obstruction, chronic airway inflammation, and airway hyperresponsiveness are essential in confirming the diagnosis. In practice, the most straightforward tests achievable in clinic are the demonstration of reversible airway obstruction by spirometry before and after bronchodilator, and the demonstration of elevated exhaled nitric oxide level as a nonspecific marker of airway inflammation. ${ }^{11}$

Features that should raise significant concern that the diagnosis of asthma may not be correct are the absence of any objective measures described herein, the absence of allergic sensitization, and the presence of a chronic wet cough. Over $85 \%$ of children with severe asthma are sensitized to one or more aeroallergens, ${ }^{10,12}$ while the presence of a chronic wet cough should alert one to the possibility of suppurative lung disease or persistent bacterial bronchitis. Table 1 lists the differential diagnoses to consider in a child with problematic severe asthma.

\section{Step 2: identify comorbidities}

Comorbidities are also identified during the initial medical assessment. Comorbidities may contribute directly to the severity of asthma, may complicate the assessment of asthma, or may be a coincidental finding.

Atopic diseases, including rhinosinusitis, dermatitis, and food allergies, are common in children with severe asthma. Inadequate treatment of rhinosinusitis is associated with worse asthma control. ${ }^{13}$ Although evidence of a direct effect of food allergies and allergic dermatitis on asthma severity is lacking, these pathologies merit optimized treatment in 
Table I Differential diagnoses and diagnostic clues to consider when assessing a child with problematic severe asthma

Differential diagnosis
Bronchiectasis
Obliterative bronchiolitis
Dysfunctional breathing
Foreign body
External airway compression, eg, vascular ring
Gastroesophageal reflux
Aspiration
Pertussis
Cystic fibrosis
Primary ciliary dyskinesia
Tracheobronchomalacia
Heart failure
Hypersensitivity pneumonitis
Diagnostic clues
Poor treatment response
Absence of any objective measures
Productive cough
Polyps
Failure to thrive
Nasal discharge from birth
Clubbing

their own right and may or may not impact asthma control. In cases where there is a risk of anaphylaxis, referral should be made to a pediatric allergy clinic where advice concerning the recognition of symptoms, allergen avoidance, and training in the use of adrenaline injections is completed. ${ }^{14}$

Obesity is a risk factor for poor asthma control, but the relationship between the two conditions is complex. Obesity likely influences asthma through a variety of pathological mechanisms including altered mechanical physiology, modified inflammatory mediator responses, and metabolic dysregulation, ${ }^{15}$ although breathlessness due to simple deconditioning is also possible. However, easily identifiable treatment of obesity remains challenging.

Symptoms of gastroesophageal reflux are often present; however, treatment does not appear to impact asthma control. In one large randomized control trial, the prescription of lansoprazole in a pediatric cohort with poorly controlled asthma had no effect. ${ }^{16}$

\section{Step 3: assess basic modifiable factors}

It is only rarely that the symptoms of asthma do not improve if an appropriate dose of inhaled steroids is deposited correctly within the airways at the correct frequency. Rectification of errors in prescription or inhaler technique may be all that is required to transform symptom control. Medication errors are identified in $48 \%$ of children with DA $;{ }^{17}$ therefore, a thorough physician and specialist nurse led review of prescribed treatments is crucial. Objective assessment of inhaler technique, including the use of an appropriate spacer device, must be undertaken as the majority of children make mistakes during assessment. ${ }^{18}$ Metered dose inhalers should always be used with a spacer device, and children should be switched to using a spacer with a mouthpiece as soon as they are able to master technique - for some children this is as young as 2-3 years. The technique required to use a dry powder device effectively is prohibitive in many cases, with over $50 \%$ of children demonstrating serious errors in technique on assessment. ${ }^{19}$ Children and families should be educated about asthma and specifically the "how", "why", and "when" of the asthma medications they are prescribed. ${ }^{7}$ Finally, all patients and carers must be given an individualized asthma action plan that details current treatment, how to recognize an exacerbation (including deterioration in peak flow values), and when to seek appropriate medical help. ${ }^{7}$ During fatal asthma attacks identified in NRAD, $45 \%$ of asthmatics had died without seeking medical advice, or before emergency medical care could be provided.

In cases where language barriers contribute to difficulties with asthma management, independent translators should be used during any clinical contact, and written advice including asthma action plans must be provided in the appropriate language.

\section{Step 4: assess complex modifiable factors Respiratory clinical nurse specialist led assessment}

In cases where control remains poor despite the abovementioned factors apparently having been addressed, the influence of more complex modifiable factors must be considered. It is this complex process that necessitates the time, expertise, and experience of a specialist MDT that is only found at tertiary referral centers. The key factor utilized to guide which members of the MDT will play the major role in a particular case is the nurse-led assessment. The findings of this assessment are discussed at an MDT meeting, and individualized plans for further evaluation and interventions decided upon.

The nurse-led assessment was first introduced at RBH in 2005, and, to date, almost 400 such assessments have been completed. The scope of the assessment has expanded since its inception and has evolved to accommodate identification of modifiable factors that were previously not well recognized. The overall aim of the assessment is to explore, in 
detail, the modifiable factors described earlier as well as find evidence of persistent allergen exposure, smoke exposure, and psychosocial factors that are contributing to poor control and determine adherence. ${ }^{17}$

The respiratory clinical nurse specialist-led assessment is divided into two parts: a hospital-based assessment and a home visit. The child and family attend a clinic appointment for the hospital-based assessment at which point the nurse specialist completes a thorough patient history with particular emphasis on environmental exposures, medications and their perceived effectiveness, adherence, and psychosocial factors. A semistructured discussion with the parent/carer incorporating multiple assessment tools (Table 2) is used to identify possible psychosocial factors that may be influencing asthma control and includes questions on home life, school, asthma treatment, and understanding of treatment. Spirometry and inhaler technique assessments are repeated. Blood tests, including a short synacthen test, total serum immunoglobulin $\mathrm{E}$ (IgE), allergen-specific IgE, and skin prick tests to a panel of aeroallergens and clinically indicated food allergens are performed. Urinary cotinine levels are measured as an objective marker of smoke exposure. ${ }^{20}$ Finally, each child is given an electronic monitoring device (EMD), which is attached to their steroid inhaler, that records the date and time of inhaler actuation for up to 12 weeks. The downloaded data provide an objective assessment of adherence.

The second part of the nurse-led assessment involves a visit to the home with the child's local community nurse if possible. The home visit represents another opportunity to identify environmental exposures and assess adherence and psychosocial issues. The home is evaluated for evidence of house dust mite exposure, pets, and mold. There is good evidence that asthma severity increases in children who are sensitized and exposed to aeroallergens such as house dust mite and pet dander. ${ }^{21}$ Advice about minimizing exposure to relevant aeroallergens is given. Evidence of smoking, such as odor and the presence of used ashtrays, is recorded. The location, quantity, and expiry date of any stored medicine are also recorded as a further assessment of likely adherence. The visit is also an opportunity to explore psychosocial issues

Table 2 A list of structured assessment tools utilized in the nurse-led assessment

Asthma Control Test/Childhood Asthma Control Test

Mini Paediatric Quality of Life Questionnaire: Mini-PAQLQ (๑ E Juniper)

Paediatric Index of Emotional Distress: PI-ED (๑ GL-Assessments)

Hospital Anxiety Depression Scale: HADS (@ GL-Assessments)

Medicines Adherence Rating Scale (๑ R Horne) in more detail, having established a relationship during the initial hospital assessment.

Additional information that assists in the formation of a comprehensive clinical and social picture is sought. The primary care physicians are contacted for information about their impression of the child and family, and a request for a list of prescriptions that have been issued over the last year is made. Further, contact with the child's school to confirm attendance rate, participation in physical education and playground activities, and to discuss how the school feel asthma affects the child's school life is arranged.

A summary of the information acquired is presented at the weekly asthma MDT meeting and enables a comprehensive picture of a child's asthma to be described to the team. The key distinction between categorizing a child as DA or STRA can be made at this point. In cases of STRA, the child will progress along a medical protocol, which includes further investigation including bronchoscopy and possible escalation of treatment. However, in the majority of cases, children fall within the DA category. For these patients, the team decides which modifiable factors that have been identified for each child case may benefit from interventions and which members of the MDT are best placed in making further assessments and planning these interventions.

Below, we describe the prominent modifiable factors that are frequently identified during the specialist nurse-led assessment and highlight the different roles of the MDT in further defining these factors and initiating treatment strategies. Often, there is significant overlap between factors and multiple members of the MDT, if not all, will have input into an individual patient's treatment.

\section{Poor adherence to maintenance therapy}

The impact of suboptimal adherence on asthma control and asthma-related morbidity is well recognized. It is associated with $80 \%$ of asthma deaths ${ }^{7}$ and is probably the most influential contributor to poor asthma control in children. ${ }^{22}$ Satisfactory adherence is defined as the administration of at least $80 \%$ of prescribed doses. $^{23}$

Accurate estimation of adherence has traditionally been very difficult. Adherence can be assessed by analyzing prescription uptake over the previous year. However, this only tells us how much medication has been collected, not how much has actually been taken.

The use of EMDs enables a more accurate assessment of adherence. These devices record electronically the date and time of actuation. ${ }^{24}$ Rates of adherence measured using this method are often inadequate and range between $30 \%$ and 
$70 \%{ }^{25}$ The children and their families know they are being monitored, and therefore adherence usually improves during this period. However, if adherence remains suboptimal, the focus needs to be on improving adherence. Those children whose control remains poor despite monitored good adherence are candidates for a step-up in treatment.

Adherence interventions to date have had disappointing results; $;{ }^{26}$ however, better understanding of patterns of adherence and reasons for poor adherence can enable us to target these interventions more effectively. For example, a child who frequently forgets may benefit from reminders and alarms, whereas one who does not take their treatment because of concerns about side effects or lack of efficacy will require an education-based approach.

In many cases, the demonstration of inadequate adherence following electronic monitoring is sufficient motivation for families and children to change their treatment behaviors. In refractory cases, our clinical psychology team are best placed to explore possible underlying factors contributing to nonadherence and influence change. Personalized plans addressing multiple facets of the problem are needed to improve adherence. ${ }^{27}$ Despite these measures, in some cases, adherence remains poor. We have utilized directly observed therapy at school to good effect; however, embarking on safeguarding proceedings may also need to be considered.

\section{Psychosocial factors}

Psychosocial factors are inextricably linked to all health issues and are of particular significance in chronic illnesses. Our experience is that the influence of psychosocial factors in problematic severe asthma is substantial and has a significant impact on other modifiable factors. In a retrospective review of the RBH staged approach to problematic severe asthma, psychosocial issues were identified as a contributory factor in $48 \%$ of cases. ${ }^{6}$

The beliefs that children and families have about asthma, the prescribed medications, or the disease will impact on how symptoms are perceived, how willing they are to engage with health advice, how adherent they may be to prescribed treatments, and how likely they are to institute suggested life changes. Some of these influencing factors may be associated with particular demographics, eg, health beliefs in particular communities; however, we have found that the assortment of contributory factors is often unique to families, and so an individualized exploration is the most appropriate strategy. Clinical psychologists can explore these beliefs, motivations, and other issues that may be directly or indirectly impacting asthma care. Evaluation and understanding of these factors allow the psychologist to assist children and families to develop practical coping strategies. Utilizing a child- and family-centered approach can enable families to better understand how to manage asthma, including improved recognition of triggers, better management of medications, improved recognition of deterioration, and when to appropriately seek further medical advice. It also results in more effective and comprehensive interventions in conjunction with the other members of the MDT, eg, in the treatment of dysfunctional breathing and addressing adherence. It is not useful to try to determine if the presence of asthma caused psychosocial issues of vice versa; if both are present, both issues should be addressed on their individual merits. Table 3 provides a brief summary of the types of psychosocial problems that our psychology team can help to identify and explore.

Psychological interventions often require a great deal of time and repeated visits before any benefit is demonstrated. Liaison with local counseling/mental health services may be required for long-term follow-up.

\section{Dysfunctional breathing}

Dysfunctional breathing is an umbrella term that includes hyperventilation syndrome, vocal cord dysfunction, and breathing pattern disorder. ${ }^{28}$ In the pediatric asthma population, dysfunctional breathing is associated with worse asthma control. ${ }^{29}$ It can be the main driver of symptoms in children with little objective evidence of lower airway pathology. In the absence of a validated screening tool in children, the prevalence of dysfunctional breathing is unknown, although one study suggests around five percent of this population experience hyperventilation syndrome. ${ }^{29}$ Experience within our MDT suggests that the specialist respiratory physiotherapist is best placed to offer assessment and treatment of breathing pattern disorders.

Table 3 Summary of some psychosocial issues that a psychology team can assess and explore

\footnotetext{
Anxiety and panic (in child and/or parent)

Depression (in child and/or parent)

Adherence

Family coping and relationships

Helping a child cooperate with medical treatments

Checking and developing the understanding of the child/family has of their illness

Life changes, eg, transferring to secondary school, desire for greater independence.

School problems, eg, bullying, poor attendance

Poor symptom perception

Difficulty in identifying and differentiating emotions and physical symptoms
} 
Children referred undergo a highly specialized clinical assessment. In cases where dysfunctional breathing is identified, an age-appropriate explanation emphasizing how abnormal breathing patterns can mimic the symptoms of asthma is an important initial step in treatment. Close collaboration between physiotherapy and psychology is particularly important for those with anxiety disorders causing hyperventilation and panic attacks. Children are taught to normalize their breathing pattern at rest through breathing control exercises, encouraging nose breathing, and appropriate use of the diaphragm. Once the child understands how to use breathing control at rest, an age-appropriate exercise and breathing pattern assessment can take place, based on play, activity, or sport. Breathing pattern re-education then continues with increasing exercise intensity. Validated measures of outcome in the pediatric population are lacking, ${ }^{30}$ making quantitative assessment of the role of breathing re-education impossible; however, it is apparent that treatment can result in long-lasting improvements in symptoms and quality of life. ${ }^{31}$

\section{Environmental exposures}

\section{Tobacco smoke}

An assessment of smoke exposure is required for all children with problematic severe asthma. Both active and passive smoking are associated with steroid resistance in children. ${ }^{32}$ In a large, nationwide study, the dramatic impact of passive smoke exposure on asthma control in children was demonstrated through a significant reduction in the rate of emergency admissions following the introduction of a smoking ban in public places. ${ }^{33}$ Therefore, eliminating exposure to smoke must be achieved before a meaningful assessment of treatment requirement can be made.

Direct evidence of exposure is obtained through measurement of urinary cotinine level. Other evidence may be obtained during the specialist nurse home visit. Identifying smoking is relatively easy in comparison to its eradication. Doctors, specialist nurses, and psychologists may all have roles to play in facilitating this difficult process as well as directing parents toward other forms of support. ${ }^{34}$

\section{Allergens}

The overwhelming majority of children with asthma are atopic, ${ }^{12}$ and those children with severe asthma are sensitized to a wider range of allergens and to a greater degree than those with mild asthma. ${ }^{10}$

Persistent exposure to allergens to which the child is sensitized is associated with increased risk of exacerbation, especially in association with viral infections ${ }^{35}$ and high levels of air pollution. ${ }^{36}$ There is little that can be done on an individual basis to reduce exposure to viruses or air pollution; however, minimizing exposure to allergens to which the child is sensitized is likely beneficial. ${ }^{21}$

All patients with DA are assessed for allergic sensitization during the initial assessment phase. The nurse-led home visit is an opportunity to identify objective evidence of allergen exposure. Practical measures, under the guidance of the specialist nurse, can be put into practice to reduce exposure to house dust mite, pet dander, and molds.

\section{Step 5: continued regular clinic follow-up and discussion at MDT meetings}

Following the identification and treatment of modifiable factors, the impact on clinical symptoms must be assessed. Improvement in lung function tests, decrease in exacerbations, and improvement in asthma control test score are important markers of improvement and are assessed at every clinic appointment. Careful attention to both basic and complex modifiable factors is required to ensure that adherence is maintained.

\section{Step 6: special cases}

\section{Two-week hospital admission}

In a small number of cases, despite input from the MDT, reported symptoms remain severe. The three possibilities in this situation are:

1. The symptoms are genuine, all modifiable factors have been addressed, and the child should actually be categorized as STRA.

2. The symptoms are genuine, but modifiable factors have not been addressed appropriately.

3. The severity of symptoms reported is not genuine.

These cases are often perplexing, the main difficulty lying in an inability to acquire an accurate and objective clinical picture. We have found that valuable objective insights that inform further management can be obtained from a two-week admission to our ward with structured MDT input.

The overall aim of the admissions is to attain a detailed MDT assessment of the factors described previously. During the admission, children attend the hospital school, participate in a daily physiotherapy-led exercise program, are taken on trips out to local parks and museums in the company of a specialist nurse, and are offered psychological input. Importantly, administration of all medications is directly observed under the supervision of a nurse, and the child is assessed medically if wheeze is reported or a request is made for reliever medication. Spirometry and exhaled nitric oxide are measured twice weekly. 
Valuable insights have been made in many children admitted to the ward for assessment, which have influenced subsequent management. These include the recognition of breathing pattern disorders, significant psychological issues, and social concerns including fabricated illness.

\section{Safeguarding}

Currently, there is little documented data on the prevalence of safeguarding issues in children with DA; however we are increasingly identifying safeguarding concerns in our clinic. In a retrospective review, safeguarding concerns were raised in $10 \%$ of our cohort.

\section{Neglect}

When DA remains uncontrolled, there is a significant risk of morbidity and/or mortality to the child. In cases where modifiable factors are not rectified sufficiently despite on-going intervention of members of the MDT, then the possibility that neglect of the child's welfare on the part of the parents must be considered.

\section{Fabricated or induced illness}

Fabricated or induced illness is suspected when the reported symptoms are inconsistent with any objective measures of asthma severity. The child is at risk of harm by being exposed to unnecessary medical investigation, interventions, and treatments or from having necessary treatments deliberately withheld. ${ }^{37}$

Safeguarding cases are often very challenging, and the experience and advice from safeguarding team is invaluable. The safeguarding team are able to offer further assessment of the difficulties within families in the context of the perceived risk to the child's health as assessed by the MDT. The team work within a framework of national legislation and guidelines pertaining to child welfare in Britain..$^{38,39}$

\section{Conclusion}

The umbrella term used to describe children with asthma that is refractory to high levels of prescribed treatment is problematic severe asthma. Within this group, the majority of children have DA; asthma that is poorly controlled because of a failure to get the basics of asthma management right. After ensuring the diagnosis is correct, the most common factor that contributes to poor asthma control is associated with medication issues. These include poor adherence to maintenance asthma therapy and inappropriate device or inhaler technique. It is essential that an objective assessment of adherence to maintenance medication is undertaken prior to any child being labeled as having severe asthma.
Subsequent modifiable reasons that may contribute to poor asthma control are harder to identify and necessitate referral to a specialist center with a pediatric DA service, incorporating an MDT. The only way to ensure DA is correctly identified and managed is within an MDT setting. A diagnosis of STRA, with associated additional investigations, and potential escalation of treatment should only be undertaken after all modifiable factors have been thoroughly addressed.

\section{Disclosure}

The authors report no conflicts of interest in this work.

\section{References}

1. Asthma UK [homepage on the Internet]. Available from: https://www. asthma.org.uk. Accessed March 20, 2016.

2. Reddel HK, Bateman ED, Becker A, et al. A summary of the new GINA strategy: a roadmap to asthma control. Eur Respir J. 2015; 46(3):622-639.

3. Lane S, Molina J, Plusa T. An international observational prospective study to determine the cost of asthma exacerbations. Respir Med. 2006;100:434-450.

4. McGeachie MJ, Yates KP, Zhou X, et al. Patterns of growth and decline in lung function in persistent childhood asthma. NEngl J Med. 2016;374(19):1842-1852.

5. Hedlin G, Bush A, Lodrup Carlsen K, et al. Problematic severe asthma in children, not one problem but many: a GA2LEN initiative. Eur Respir J. 2010;36(1):196-201.

6. Bracken M, Fleming L, Hall P, et al. The importance of nurse-led home visits in the assessment of children with problematic asthma. Arch Dis Child. 2009;94(10):780-784.

7. Levy ML. The national review of asthma deaths: what did we learn and what needs to change? Breathe (Sheff). 2015;11(1):14-24.

8. Nasser S. An imperfect "PAST" lessons learned from the National Review of Asthma Deaths (NRAD) UK. Respir Res. 2016;17:87.

9. Chung KF, Wenzel SE, Brozek JL, et al. International ERS/ATS guidelines on definition, evaluation and treatment of severe asthma. Eur Respir J. 2014;43(2):343-373.

10. Sharples J, Gupta A, Fleming L, et al. Long-term effectiveness of a staged assessment for paediatric problematic severe asthma. Eur Respir J. 2012;40(1):264-267.

11. Woo SI, Lee JH, Kim H, Kang JW, Sun YH, Hahn YS. Utility of fractional exhaled nitric oxide (F(E)NO) measurements in diagnosing asthma. Respir Med. 2012:106(8):1103-1109.

12. Bossley CJ, Fleming L, Gupta A, et al. Pediatric severe asthma is characterized by eosinophilia and remodeling without $\mathrm{T}(\mathrm{H}) 2$ cytokines. J Allergy Clin Immunol. 2012;129(4):974-982.

13. Deliu M, Belgrave D, Simpson A, Murray CS, Kerry G, Custovic A. Impact of rhinitis on asthma severity in school-age children. Allergy. 2014;69:1515-1521.

14. Royal College Paediatrics and Child Health. Allergy Care Pathways for Children Food Allergy. London, UK: RCPCH; 2011.

15. Vijayakanthi N, Grelly J, Rastogi D. Pediatric obesity-related asthma: the role of metabolic dysregulation. Pediatrics. 2016;137(5):1-16.

16. American Lung Association Asthma Clinical Research Centers; Holbrook JT, Wise RA, Gold BD, et al. Lansoprazole for children with poorly controlled asthma: a randomized controlled trial. JAMA. 2012; 307(4):373-380.

17. Bracken M, Fleming L, Hall P, et al. The importance of nurse-led home visits in the assessment of children with problematic asthma. Arch Dis Child. 2009;94(10):780-784.

18. Alexander DS, Geryk L, Arrindell C, et al. Are children with asthma overconfident that they are using their inhalers correctly? J Asthma. 2016;53(1):107-112. 
19. Westerik JA, Carter V, Chrystyn H, et al. Characteristics of patients making serious inhaler errors with a dry powder inhaler and association with asthma-related events in a primary care setting. J Asthma. 2016;53(3): 321-329.

20. Wang Y, Yang M, Tian L, et al. Relationship between caregivers' smoking at home and urinary levels of cotinine in children. Int J Environ Res Public Health. 2014;11(12):12499-12513.

21. Gent JF, Kezik JM, Hill ME, Tsai E, Li DW, Leaderer BP. Household mold and dust allergens: exposure, sensitization and childhood asthma morbidity. Environ Res. 2012;118:86-93.

22. Lindsay JT, Heaney LG. Non-adherence in difficult asthma and advances in detection. Expert Rev Respir Med. 2013;7(6):607-614.

23. Santos PM, D’Oliveira A, Noblat A, et al. Predictors of adherence to treatment in patients with severe asthma treated at a referral center in Bahia, Brazil. J Bras Pneumol. 2008;34(12):995-1002.

24. Pilcher J, Shirtcliffe P, Patel M, et al. Three-month validation of a turbuhaler electronic monitoring device: implications for asthma clinical trial use. BMJ Open Respir Res. 2015;2(1):e000097.

25. Eakin MN, Rand CS. Improving patient adherence with asthma selfmanagement practices: what works? Ann Allergy Asthma Immunol. 2012; 109(2):90-92.

26. Engelkes M, Janssens HM, de Jongste JC, et al. Medication adherence and the risk of severe asthma exacerbations: a systematic review. Eur Respir J. 2015;45:396-407.

27. Klok T, Kaptein AA, Duiverman EJ, Brand PL. Long-term adherence to inhaled corticosteroids in children with asthma: observational study. Respir Med. 2015;109(9):1114-1119.

28. Barker N, Jones M, O’Connell NE, Everard ML. Breathing exercises for dysfunctional breathing/hyperventilation syndrome in children. Cochrane Database Syst Rev. 2013:18;(12):CD010376.
29. De Groot E, Duiverman E, Brand PL. Dysfunctional breathing in children with asthma: a rare but relevant comorbidity. Eur Respir J. 2013:41; 1068-1073.

30. Macedo T, Freitas D, Chaves G, Holloway E, Mendonça KM. Breathing exercises for children with asthma. Cochrane Database Syst Rev. 2016;12(4).

31. Barker N, Everard M. Breathing retraining as a treatment modality for dysfunctional breathing in children. Eur Respir J. 2014;44(Suppl 58):4680.

32. Kobayashi Y, Bossley C, Gupta A, et al. Passive smoking impairs histone deacetylase-2 in children with severe asthma. Chest. 2014;145(2):305-312.

33. Mackay D, Haw S, Ayres JG, Fischbacher C, Pell JP. Smoke-free legislation and hospitalizations for childhood asthma. $N$ Engl J Med. 2010;363(12):1139-1145.

34. Stead LF, Koilpillai P, Lancaster T. Additional behavioural support as an adjunct to pharmacotherapy for smoking cessation. Cochrane Database Syst Rev. 2015(10):CD009670.

35. Murray CS, Poletti G, Kebadze T, et al. Study of modifiable risk factors for asthma exacerbations: virus infection and allergen exposure increase the risk of asthma hospital admissions in children. Thorax. 2006;61(5):376-382.

36. Cakmak S, Dales RE, Coates F. Does air pollution increase the effect of aeroallergens on hospitalization for asthma? J Allergy Clin Immunol. 2012;129(1):228-231.

37. Royal College Paediatrics and Child Health. Fabricated or Induced Illness by Carers: A Practical Guide for Paediatricians. London, UK: RCPCH; 2009.

38. HM Government. Working Together to Safeguard Children: A Guide to Interagency Working to Safeguard Children. London, UK: The Stationery Office; 2006.

39. UK Legislation. Children's Act 2004. Available from: http://www. legislation.gov.uk/ukpga/2004/31/contents. Accessed March 20, 2016.
Journal of Asthma and Allergy

\section{Publish your work in this journal}

The Journal of Asthma and Allergy is an international, peer-reviewed open access journal publishing original research, reports, editorials and commentaries on the following topics: Asthma; Pulmonary physiology; Asthma related clinical health; Clinical immunology and the immunological basis of disease; Pharmacological interventions and

\section{Dovepress}

new therapies. This journal is included in PubMed. The manuscript management system is completely online and includes a very quick and fair peer-review system, which is all easy to use. Visit http://www dovepress.com/testimonials.php to read real quotes from published authors. 